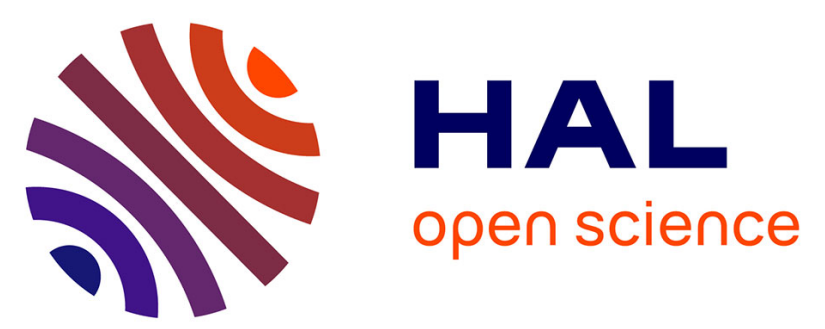

\title{
Abnormal vascularization of soft-tissue sarcomas on conventional MRI: Diagnostic and prognostic values
}

Pauline Ledoux, Michèle Kind, François Le Loarer, Eberhard Stoeckle, Antoine Italiano, Franck Tirode, Xavier Buy, Amandine Crombé

\section{To cite this version:}

Pauline Ledoux, Michèle Kind, François Le Loarer, Eberhard Stoeckle, Antoine Italiano, et al.. Abnormal vascularization of soft-tissue sarcomas on conventional MRI: Diagnostic and prognostic values. European Journal of Radiology, 2019, 117, pp.112 - 119. 10.1016/j.ejrad.2019.06.007 · hal-03486081

\section{HAL Id: hal-03486081 \\ https://hal.science/hal-03486081}

Submitted on 20 Dec 2021

HAL is a multi-disciplinary open access archive for the deposit and dissemination of scientific research documents, whether they are published or not. The documents may come from teaching and research institutions in France or abroad, or from public or private research centers.
L'archive ouverte pluridisciplinaire HAL, est destinée au dépôt et à la diffusion de documents scientifiques de niveau recherche, publiés ou non, émanant des établissements d'enseignement et de recherche français ou étrangers, des laboratoires publics ou privés.

\section{(ㄷ)(1) $\$$}

Distributed under a Creative Commons Attribution - NonCommerciall 4.0 International 


\title{
Abnormal Vascularization of Soft-Tissue Sarcomas on Conventional MRI: Diagnostic and Prognostic Values
}

\author{
Pauline Ledoux ${ }^{1}$, Michèle Kind ${ }^{1}$, François Le Loarer ${ }^{2,3,4}$, Eberhard Stoeckle ${ }^{5}$, Antoine \\ Italiano $^{3,4,6}$, Franck Tirode ${ }^{7}$, Xavier Buy ${ }^{1}$, Amandine Crombé ${ }^{1,3,4}$,
}

1. Department of Radiology, Institut Bergonié, Comprehensive Cancer Center, F-33076, Bordeaux, France

2. Department of Pathology, Institut Bergonié, Comprehensive Cancer Center, F-33076, Bordeaux, France

3. University of Bordeaux, F-33000, Bordeaux

4. INSERM U1218, Action, Institut Bergonié, Bordeaux, France

5. Department of Surgery, Institut Bergonié, Comprehensive Cancer Center, F-33076,

Bordeaux, France

6. Department of Medical Oncology, Institut Bergonié, Comprehensive Cancer Center, F33076, Bordeaux, France

7. Cancer Research Center of Lyon, University of Lyon, Université Claude Bernard Lyon 1, CNRS 5286, INSERM U1052, Lyon.

Corresponding author: Dr Amandine Crombé, MD

Email: a.crombe@bordeaux.unicancer.fr

Tel: +33 (0) 56333333

Fax: +33 (0) 56333330

Address: Department of Radiology, Institut Bergonié, 229, cours de l'Argonne, F-33076, Bordeaux cedex 


\title{
Abnormal Vascularization of Soft-Tissue Sarcomas on Conventional MRI: Diagnostic and Prognostic Values
}

\begin{abstract}
Purpose: To assess the prevalence of abnormal vessels inside and surrounding soft-tissue sarcomas (STS) on conventional MRIs so as to evaluate their correlations with particular histotypes, histological grades, and prognosis.

Method: This single-center retrospective study included 157 adult patients (median age: 61) with histologically-proven non-metastatic STS. All had pre-treatment conventional contrastenhanced MRI. Two radiologists reported: presence of abnormal flow-voids, number and distribution (peri-tumoral and/or intra-tumoral), percentage of tumor circumference it covered, maximal diameter. The radiological findings were correlated with histopathology. Associations were evaluated with Chi-2 or t-tests. Survival analysis (for metastasis-free survival (MFS) and overall survival (OS)) included log-rank tests and multivariate Coxmodel.
\end{abstract}

Results: Twenty-nine of 157 (18.5\%) STS showed abnormal flow-voids that were peritumoral $(9 / 157,5.7 \%)$, intra-tumoral $(5 / 157,3.2 \%)$ or both intra- and peri-tumoral $(15 / 157$, 9.6\%). Ten STS had more than 5 flow-voids, all being grade II-III, namely: 4 undifferentiated sarcomas, 2 solitary fibrous tumors, 1 alveolar soft-part sarcoma (ASPS), 2 leiomyosarcomas and 1 pleomorphic liposarcoma. The distribution of flow-voids was associated with survivals in the univariate analysis: patients with abnormal peritumoral flow-voids (APTFV) showed poorer OS and MFS ( $\mathrm{p}=0.039$ and 0.014 , respectively). These associations did not remain significant in multivariate analysis. Radio-pathological correlations revealed large tortuous tumoral neo-vessels with intra-vascular thrombi of tumor cells in ASPS and in one case of undifferentiated sarcoma displaying enrichment in genes involved in neo-angiogenesis at transcriptional level. 
Conclusions: APTFV on conventional MRIs may be associated with a higher risk of metastatic relapse and poorer OS in STS patients.

\section{Keywords}

Magnetic Resonance Imaging/MRI;

Soft tissue sarcoma;

Soft tissue tumor;

Flow-voids;

Vascularization;

Prognosis

\section{Highlights}

1. Abnormal tumoral vessels on MRI mostly occur in grade II-III STS.

2. Abnormal tumoral vessels are a hallmark of ASPS and solitary fibrous tumors.

3. Abnormal tumoral vessels are not helpful to discriminate other STS histotypes.

4. APTFV may correlate with poorer OS due to the higher risk of metastatic relapses.

\section{Abbreviations}

APTFV: abnormal peri-tumoral flow-voids

ASPS: Alveolar soft-part sarcoma

CE: Contrast enhanced

CI95\%: 95\% confidence interval

CT: computed tomography

DCE-MRI: Dynamic contrast enhanced MRI

FNCLCC: Federation Nationale des Centres de Lutte Contre le Cancer

HES: Hematoxyline and eosin stained slices

HR: Hazard ratio

ICC: Inter-class correlation coefficient

k: Cohen's kappa

LRFS: Local relapse-free survival

MFS: Metastasis-free survival

OS: Overall survival 
ssGSEA: single sample gene set enrichment analysis

SFT: Solitary fibrous tumor

STS: Soft-tissue sarcoma

VEGF: Vascular endothelial growth factor

WI: Weighted imaging

wk: weighted kappa 


\section{Introduction}

Soft-tissue sarcomas (STS) are a heterogeneous group of ubiquitous malignant mesenchymal tumors. When located in the soft-tissues of the trunk wall and extremities, clinical guidelines recommend to perform MRIs for local staging [1]. MRI analyses of STS should typically include the evaluation of the size, depth, location, tumor matrix, margins and surrounding tissues. However, the vascularization patterns of STS have been poorly investigated.

Tumor vascularization can be analyzed with MR-angiography or dynamic-contrast enhanced MRIs (DCE-MRI) but can also be captured on conventional MRIs as flow-voids, that is to say vessels with signal loss especially on spin echo imaging with long echo time [2,3]. Some STS histotypes are known to harbor abnormal vascular patterns on MRIs. Alveolar soft-part sarcomas (ASPS) and solitary fibrous tumors (SFT) can demonstrate large tortuous intra- and peri-tumoral flow-voids [4-9]. Interestingly, pro-angiogenic factors, such as vascular endothelial growth factors (VEGF) and their receptors, are strongly expressed in ASPS and SFT, which correlate with their rich vascular networks on microscopy. Therefore, the presence of flow-voids on MRIs may be an indicator of strong neo-angiogenesis in STS [1012]. In keeping with this hypothesis, clear cell renal carcinoma and their metastases, which are known to display both high expression of pro-angiogenic factors and abundant vasculature, display flow-voids on MRIs [13].

Besides SFT and ASPS, previous studies have shown high VEGF expression in epitheloid sarcomas, undifferentiated pleomorphic sarcomas (UPS), dermatofibrosarcomas protuberans and leiomyosarcomas [14,15]. Associations between pro-angiogenic factors, histological grade (the strongest prognostic factor for STS) and patients' outcomes have also been reported [15-18]. If a correlation does exist between the expression of pro-angiogenic molecules, hypervascular patterns on MRIs and patients' outcomes, one could consider the 
MRI as a tool to identify STS with both an abnormal vasculature and a potentially greater sensitivity to anti-angiogenic regimens.

Thus, the aims of this exploratory retrospective study were to investigate the prevalence of abnormal intra- and peritumoral flow-voids on conventional MRIs of STS, and to evaluate their associations with histological grading, particular histotypes and patients' outcomes.

\section{Material and methods}

\subsection{Study Design}

Our institutional review board approved this single-center study and the need for informed consent was waived by its retrospective nature. All consecutive adult patients addressed to our sarcoma reference center between January 2008 and June 2016 were included when they presented with: histologically-proven STS, tumors located in the trunk wall or extremities, no metastasis at baseline on chest CT-scans at least, available pre-treatment contrast-enhanced MRIs, and available histopathological samples of the surgical specimen. The patients with well-differentiated liposarcoma, angiosarcoma or extra-osseous bone sarcoma were excluded. The following clinical and pathological data were retrieved from medical reports: gender, age, World Health Organization performance status, histotype, histological grading (according to the French Federation National des Centres de Lutte contre le Cancer [FNCLCC] grading system on initial microbiopsy in case of neo-adjuvant treatments or surgical specimen otherwise [16]), tumor location, chemotherapy and/or radiotherapy (neo-adjuvant or adjuvant), local relapse, metastatic relapse and death from any cause. At our institution, routine follow-ups consist in clinical examinations and chest radiographs every 3 months for 2 years, every 6 months for 5 years and then annually up until 10 years after surgery. These examinations were complemented by chest-computed tomography (with 1mm-thick reconstruction) and MRIs for local evaluation in case of abnormal or doubtful findings, in 
agreement with last recommendations of the European Society of Medical Oncology [1]. All relapses were histologically proven.

\subsection{MRI acquisition}

Examinations were carried out on 1.5-Tesla magnets from different radiological centers. Indeed, some patients underwent an initial MRI in external radiological centers before they had been addressed to our regional sarcoma reference center. Spin echo T2-weighted imaging (-WI), T1-WI, fat-suppressed contrast enhanced (CE) T1-WI (gradient echo or spin echo sequence) and at least two orthogonal acquisition plans were available for all patients. Section thickness ranged from 3 to $5 \mathrm{~mm}$. Coils, field-of-views and matrices were adapted to tumor sizes and locations. Ranges of repetition time/echo time were: $500-700 / 10-15 \mathrm{msec}$ for T1-WI and 2400-4500/100-130msec for T2-WI.

\subsection{MRI analysis}

All MRIs were anonymized and independently reviewed on a picture archiving and communication system (Enterprise Imaging, AGFA, the Netherland) by two radiologists: a senior radiologist from a sarcoma reference center and a fellow with 3 years of experience in MRIs including 6 months in a sarcoma reference center in order to assess inter-observer agreement. A final consensual reading was made 2 months after the first readings and used for the statistical analysis.

First, the radiologists reported: (1) the presence of abnormal flow-voids (defined as abnormal, non-anatomical, serpiginous or linear structures with low SI on T1-WI and T2-WI possibly in communication with feeder vessels). When present, they analyzed: (2) their distribution (categorized as intra-tumoral or peri-tumoral or both); (3) their count (categorized as 0 or 1-5 or 6-10 or $>10$ ), (4) the tumor circumference covered by peri-tumoral flow-voids (categorized 
as $0 \%$ or $1-25 \%$ or $>25 \%$ ) and (5) their maximal diameter. In addition, the following radiological variables were reported: the longest diameter of the STS and the MRI growth pattern on the whole tumor circumference on T2-WI and CE-T1-WI (categorized as: 'pushing-type' when the tumor was entirely well-defined, 'focal-type' and 'diffuse type' when irregular borders and infiltration of surrounding tissues represented $<25 \%$ and $\geq 25 \%$ of tumor circumference, respectively) [19].

\subsection{Histopathological and molecular analyses}

The hematoxyline and eosin stained (HES) slices of the surgical specimen of STS patients with abnormal vasculature on MRIs were collected and retrospectively analyzed by a senior pathologist with expertise in STS, blinded to the radiological results. The pathologist reported on the tumors that showed: (i) an usual vasculature regarding its histotype, or (ii) an increased number of vessels without abnormal morphology, or (iii) an increased number of vessels with unusual architecture. These latter cases were analyzed by tumor RNA sequencing from formol-fixed paraffin-embedded tumor samples to evaluate the enrichment in genes involved in angiogenesis. Total RNA was extracted from formalin-fixed paraffin-embedded tissue section using the Formapure RNA kit (Beckman Coulter, Brea, California) following the manufacturer's recommendations. Quantity and quality of total RNA were evaluated using NanoDrop (Thermo Fisher Scientific) and Tape Station with Hs RNA Screen Tape (Agilent) using a cut off of $\mathrm{DV}_{200}$ (defined as the percentage of RNA fragments above 200 nucleotides) above $13 \%$. Libraries were prepared with 100 ng of total RNA using TruSeq RNA Access Library Prep Kit (Illumina, San Diego, USA). Libraries were pooled by groups of 12 samples. Paired-end sequencing was performed using the NextSeq 500/550 High Output V2 kit (150 cycles) on Illumina NextSeq 500 platform (Illumina, San Diego, CA). 
Sequencing data (65 million reads per sample on average) were aligned with STAR on GRCh 38 reference genome as previously reported (ref PMID: 30407212) [20]. A single sample gene set enrichment analysis (ssGSEA) was performed in order to identify whether the tumor sample was enriched in angiogenic genes signature from the "hallmark_angiogenesis gene sets collection" from the Molecular Signatures DataBase [21-23].

\subsection{Statistical analysis}

Associations between categorical and/or ordinal variables were tested with Chi-2 or Fisher exact tests when appropriate. Continuous variables were compared using the Wilcoxon or Student test depending on the Shapiro-Wilk normality test.

Inter-observer agreements were assessed using the interclass correlation coefficient with the two-way mixed model (ICC), Cohen's Kappa (k) and weighted Kappa (wk) for continuous, dichotomized and ordinal variables, respectively. The agreement for ICC was defined as good $(>0.75)$, moderate $(0.5-0.75)$ or poor $(<0.5)$. The agreement for $\mathrm{k}$ and $\mathrm{w}-\mathrm{k}$ was defined as slight (0-0.20), fair (0.21-0.40), moderate (0.41-0.60), substantial (0.61-0.80) and almost perfect $(0.81-0.99)[24]$.

Overall survival (OS), local relapse-free survival (LRFS) and metastasis-free survival (MFS) were defined as the time lapse between curative surgery and death (from any cause), local relapse or metastatic relapse, respectively. MFS, LRFS and OS were estimated using the Kaplan-Meier method. Comparisons of survival curves depending on radiological variables were performed with the Log-rank test. Multivariate Cox models were built to determine the associations between the radiological features and the different survival indicators adjusted by age, gender, performance status, FNCLCC grade, longest diameter (categorized as $<5 \mathrm{~cm}$ or $\geq 5 \mathrm{~cm}$ ), MRI growth-patterns, tumor depth, surgical margins, chemotherapy and radiotherapy. For patients who were event free at the time of the analysis, survivals were censored at the 
date of final contact for living patients. Patients with missing value(s) and/or with a postsurgery follow-up of less than 2 years without event were removed from the survival analyses. All tests were two-tailed. Statistical analyses were done using the SPSS statistical package (version 21.0, IBM). A p-value <0.05 was deemed significant.

\section{Results}

\subsection{Patients}

Table 1 summarizes the epidemiological characteristics of the study population. Of the 157 included patients, $69(43.9 \%)$ were women. Median age was 61 years old (range: 15-92, mean: $59.1 \pm 17.7)$. Mean size was $89 \pm 56 \mathrm{~mm}$. The most common histotype was undifferentiated pleomorphic sarcoma (UPS) (39/157 (24.8\%)), followed by myxofibrosarcoma (24/157 (15.3\%)) and leiomyosarcoma (16/157 (10.2\%)), respectively.

\subsection{Associations between STS vascularization on conventional MRIs, grade and histotypes}

In total, 29/157 (18.5\%) tumors demonstrated abnormal flow-voids on MRIs, which were peri-tumoral in 9 cases, intra-tumoral in 5 cases and both intra- and peri-tumoral in 15 cases. There were 21/157 (13.4\%) grade I, 48/157 (30.6\%) grade II and 88/157 (56.1\%) grade III STS. Table 2 shows the assessment of associations between MRI evaluation of STS vascularization and histological grade. There was no significant association between the flowvoids' presence, number, distribution, diameter or covered surface. None of the grade I STS showed peri-tumoral flow-voids. However, the tumors with more than 5 flow-voids $(n=10)$, were all grade II or III. These tumors were: SFT $(n=2)$, ASPS $(n=1)$, pleomorphic liposarcoma $(n=1)$, leimyosarcoma $(n=2)$, undifferentiated sarcoma $(n=4)$.

The inter-observer agreements of the MRI features are given in table 3. All showed at least moderate reproducibility. 


\subsection{Radiological-histopathological correlations}

Retrospective reviews of HES slices of STS with abnormal vasculature on MRIs showed a prominent vascular pattern with large confluent abnormal vessels in 4 cases: 2 SFT (Fig. 1A), 1 ASPS (Fig. 1B) and 1 undifferentiated sarcoma with an epithelioid phenotype (previously reported as a UPS) (Fig. 2). Vascular invasions by tumor cells were seen in the samples of ASPS and undifferentiated sarcomas. The latter sample also included large vessels circumscribing tumor sheets at their periphery. In this case, ssGSEA showed strong upregulation of genes involved in neo-angiogenesis with a normalized enrichment score of 4.18 (with a false discovery rate q-value of 0.0001) (Fig. 2C). In the other cases, vessels did not demonstrate abnormal morphologies but were slightly more numerous than expected and seemed either recruited by or trapped in the borders of the tumor (Fig. 3).

\subsection{Prognostic value of STS vascularization on conventional MRIs}

Three patients were removed from the survival analyses because of early loss of follow-up. Mean LRFS, MFS and OS of the series were: 89 months (CI95\%=[82-96]), 80 months $(\mathrm{CI} 95 \%=[73-88])$ and 87 months $(\mathrm{CI} 95 \%=[80-93])$, respectively. There were 35 local relapses, 49 metastatic relapses and 43 deaths. Table 4 summarized the univariate survival analyses. None of the flow-voids-related features were associated with local relapses. However, the distribution of flow-voids was significantly associated with MFS and OS in the univariate analysis (log-rank p-value $=0.014$ and 0.039 , respectively). STS with abnormal peritumoral flow-voids (APTFV) had poorer MFS (35 months, CI95\%=[11-59] versus 89 months, CI95\%=[75-90]) and OS (43 months, CI95\%=[18-68] versus 89 months, CI95\%=[82-95])

(Fig. 4). In the multivariate analysis, APTFV did not remain an independent prognostic factor for PFS and OS after adjustments with potential cofounders (hazard ratio $(\mathrm{HR})=2.0$, 
$\mathrm{CI} 95 \%=[0.8-5.6], \mathrm{p}=0.116$, and $\mathrm{HR}=2.2, \mathrm{CI} 95 \%=[0.9-7.6], \mathrm{p}=0.096$, respectively). A significant association was found between the MRI infiltrative growth pattern co-variable and the presence of APTFV ( $\mathrm{p}=0.040)$.

\section{Discussion}

The vascularization pattern of STS on imaging has been poorly investigated. In this study, we reported the prevalence of abnormal intra- and peri-tumoral flow-voids on (turbo) spin echo MRI sequences in a large cohort of STS located on the trunk wall and extremities, as a result of 8 years of activity of one sarcoma reference center. We found that flow-voids were rather infrequent and prevailed in FNCLCC grade II and III STS. Radio-pathological correlations demonstrated atypical, enlarged, confluent tumor vessels in 4 cases, including one atypical undifferentiated clear cell sarcoma with an unusual pro-angiogenic transcriptomics profile. In the univariate analysis, APTFV were associated with lower OS and MFS, even they if they did not remain statistically associated with these outcomes in the multivariate analysis.

Our results concur with the few available data regarding STS vascularization on MRIs. Indeed, all the SFT and ASPS in our series showed unusual vasculature on MRIs as already observed [4-9]. Herein, we also showed that other tumor histotypes could demonstrate flowvoids, notably leiomyosarcoma, pleomorphic liposarcoma and undifferentiated sarcomas. This suggests that the presence of flow-voids inside and around a soft-tissue tumor alone cannot help diagnose SFT or ASPS. In addition, it should be noted that flow-voids have already been described in benign soft-tissue tumors or pseudo-tumors, such as schwannomas, desmoid tumors, hemangiomas, or arteriovenous malformations [25-28]. However, except for the patient with the atypical undifferentiated clear cell sarcoma, it should be noted that flow-voids were less numerous or dystrophic in these other STS histotypes compared to SFT and ASPS. 
The histopathological analysis confirmed this impression because the flow-voids corresponded to normal vessels that were recruited by the tumor rather than tumoral neovessels. Conversely, endovascular thrombi of tumor cells were only seen in ASPS and the atypical undifferentiated clear cell sarcoma, suggesting an abnormal permeability of tumoral neo-vessels in these cases.

Though rarely observed, APTFV were associated with worse MFS and OS in the univariate analysis. The results were not significant in the multivariate analysis most likely because of the lack of statistical power and confusion bias with other variables, notably MRI growth patterns and FNCLCC grade, which is the strongest STS prognostic factor [17, 19]. Indeed, all patients with APTFV had grade II or III STS. Furthermore, we found a significant association between the infiltrative growth pattern and the presence of APTFV. Nakamura et al. found that the MRI growth pattern was an independent prognostic factor of disease-free survival and metastasis-free survival [19].

To explain this association with patients' outcomes in the univariate analysis, it could be hypothesized that APTFV may favor the occurrence of distant hematogenous metastases through the formation of endovascular thrombi of tumor cells, as it was observed on the HES slices of ASPS and the atypical undifferentiated clear cells sarcoma. Regarding ASPS, Setsu et al. identified a specific underlying phenomenon called "invasion-independent" mechanism [29]. It consists in the wrapping of clusters of tumor cells by endothelial cells that spread in the tumor's vascular channels. This could explain the high rate of metastasis in ASPS patients, ranging from 50 to $79 \%[4,30]$. However, this mechanism has never been shown in other STS.

Besides vascularization patterns on MRIs, a common point between ASPS and SFT in general, and the atypical undifferentiated clear cell sarcomas from our series is the high 
expression rate of pro-angiogenesis-related genes. Previous studies showed recurrent overexpressed angiogenesis-promoting genes [12,30] explaining the efficacy of tyrosinekinase inhibitors for ASPS and SFT [12, 30-32]. Though progression-free survival improved, the OS in the sole phase 3 of placebo-controlled clinical trials (which evaluated an antiangiogenic molecule (i.e. pazopanib) in metastatic STS patients) did not. It should be noted that the inclusion of patients was not based on the expression of pro-angiogenic molecules such as VEGF-receptors of platelet-derived growth factors receptors [33]. Further trials which therapeutic strategy would be based on the expression of pro-angiogenic factors could validate the suspected association between abnormal vascularization on the MRI and the expression of pro-angiogenic factors. Thus, MRIs could help better select STS patients eligible for anti-angiogenic treatments.

Our study has several limits. First, this is a retrospective single-center study. Few tumors showed abnormal vasculature on MRIs leading to a lack of statistical power in the survival analysis. The retrospective nature of the study was responsible for heterogeneous MRI acquisition protocols. Most patients were referred to our sarcoma reference center with a basic conventional MRI performed in non-specialized radiological centers. Consequently, we limited our analysis to qualitative or semi-quantitative radiological features. Advanced imaging techniques could have brought quantitative information about tumor vascularization, notably DCE-MRI.

Second, we limited the pathological analysis to a retrospective review of HES slices with a qualitative assessment. RNA sequencing was restricted to atypical cases of tumoral neovessels regarding the reported histotype. This was only found in one patient with high-grade undifferentiated clear cell sarcoma. We did not perform the GSEA on all the tumors of the series, and neither did we compare the expression of pro-angiogenic genes between tumors 
with flow-voids and tumors without any because it was beyond the scope of our pilot study. Consequently, it is possible that patients with high levels of expression of pro-angiogenic genes may not exhibit abnormal vascularization on conventional MRIs. A next step would be to correlate the MRI vascular phenotype with its genotype in a prospective study. Other techniques for analyzing STS vascularization could be evaluated in further studies, such as 2D or 3D quantitative intra-tumoral micro-vessel density (and, by analogy, peri-tumoral) with the help of various immunohistochemistry targeting endothelial antigens [34].

Furthermore, our series did not include some STS histotypes, notably dermatofibrosarcoma protuberans or angiosarcomas, which are known for possibly harboring deregulated proangiogenic pathways involving VEGF and platelet-derived growth factors receptors, respectively $[35,36]$. Indeed, both histotypes are rarely explored with MRIs because of their usual anatomical locations (in superficial tissues for dermatofibrosarcoma, and visceral or superficial for angiosarcoma). Moreover, we did not include well-differentiated liposarcomas because their main diagnostic challenge is the distinction with benign lipomas. We also excluded extra-osseous bone sarcomas because their grading system and prognostic factors are those of bone sarcomas.

To conclude, this retrospective exploratory study illustrates the rarity of abnormal vascularization of STS on conventional MRIs despite the fact that abnormal enlarged flowvoids, especially at the borders of a sarcoma, could indicate a tumor with a poorer prognosis that possibly expresses targetable pro-angiogenic factors. 


\section{FIGURES AND TABLES}

Table 1. Epidemiological characteristics of the study population

Table 2. Association between abnormal vascularization on conventional MRIs and histological grading

Table 3. Inter-observer agreement of the features that evaluate the vascularization of STS on conventional MRIs.

Table 4. Univariate survival analysis

Figure 1. Radio-pathological correlations of solitary fibrous tumors and alveolar soft part sarcoma with abnormal vasculature on both imaging and histological samples. (A.1) A 50year-old woman presented with a deep-seated alveolar soft-part sarcoma of the left thigh that showed abnormal intra- and peri-tumoral flow-voids on spin echo MRI sequences (white arrows). (A.2) The corresponding hematoxyline and eosin stained slices demonstrated abundant vascularity associating enlarged abnormal vascular channels and numerous capillaries (black arrows), in which cluster of tumor cells were seen (black arrow head in the magnification at the right upper corner). (B.1) A 60-year-old woman presented with a deep solitary fibrous tumor of the popliteal region. The MRI showed tortuous and serpiginous vessels (ie, flow-voids) that predominated around the tumor (white arrows), which correlated with the histological findings (B.2). Abbreviations: Ax: axial, Coro: coronal, Fat Sat: fat saturation, Sag: sagittal, STIR: short inversion time recovery, WI: weighted-imaging.

Figure 2. A 60-year-old man presented with a deep-seated, high-grade, undifferentiated clear cell sarcomas of the left thigh (A) with a striking abundant intra- and peri-tumoral vascularization (white arrows). Histological analysis (B) of the surgical specimen (after neoadjuvant cytotoxic chemotherapy) showed a poor response to anthracycline ( $70 \%$ residual viable cells) and confirmed an atypical dystrophic vascularization (black arrows) with endovascular tumoral spreading (* and black arrow head on the magnification at the left upper corner). (C) Single sample Gene Set Enrichment Analysis (ssGSEA) showed a strong overrepresentation at the top of a ranked list (or ordered) list of genes involved in angiogenesis from the collection of the Molecular Signatures DataBase. Abbreviations: Ax: 
axial, Coro: coronal, ES: enrichment score, Fat Sat: fat saturation, FDR q-val: false discovery rate q-value; NES: normalized enrichment score; Sag: sagittal, STIR: short TI inversion recovery, WI: weighted-imaging,

Figure 3. Intra- and peri-tumoral flow-voids without atypical findings on histological samples. Patient presented with a deep-seated, high-grade undifferentiated pleomorphic sarcomas of the left thigh that exhibited peritumoral enhancement and ill-defined margins on fat sat contrast-enhanced T1-weighted imaging (A). (B) On turbo spin echo T2-weithed imaging, numerous small intra- and peri-tumoral flow voids were seen (white arrows). (C) On hematoxyline and eosin stained slices, these vessels were indeed more numerous as expected but their morphology was not atypical regarding the histotype (black arrowheads for intratumoral vessels and black arrows for peri-tumoral vessels).

Figure 4. Kaplan-Meier survival curves for metastasis free survival (A) and overall survival (B) depending on the presence of abnormal peritumoral flow-voids (APTPFV). 


\section{References}

[1] P.G. Casali, N. Abecassis, S. Bauer, R. Biagini, S. Bielack, S. Bonvalot, I. Boukovinas, J.V.M.G. Bovee, T. Brodowicz, J.M. Broto, A. Buonadonna, E. De Álava, A.P. Dei Tos, X.G. Del Muro, P. Dileo, M. Eriksson, A. Fedenko, V. Ferraresi, A. Ferrari, S. Ferrari, A.M. Frezza, S. Gasperoni, H. Gelderblom, T. Gil, G. Grignani, A. Gronchi, R.L. Haas, A. Hannu, B. Hassan, P. Hohenberger, R. Issels, H. Joensuu, R.L. Jones, I. Judson, P. Jutte, S. Kaal, B. Kasper, K. Kopeckova, D.A. Krákorová, A. Le Cesne, I. Lugowska, O. Merimsky, M. Montemurro, M.A. Pantaleo, R. Piana, P. Picci, S. Piperno-Neumann, A.L. Pousa, P. Reichardt, M.H. Robinson, P. Rutkowski, A.A. Safwat, P. Schöffski, S. Sleijfer, S. Stacchiotti, K. Sundby Hall, M. Unk, F. Van Coevorden, W. Van der Graaf, J. Whelan, E. Wardelmann, O. Zaikova, J.Y. Blay, ESMO Guidelines Committee and EURACAN, Soft tissue and visceral sarcomas: ESMO-EURACAN Clinical Practice Guidelines for diagnosis, treatment and follow-up, Ann. Oncol. Off. J. Eur. Soc. Med. Oncol. 29 (2018) iv51-iv67. doi:10.1093/annonc/mdy096.

[2] D. Chien, A. Goldmann, R.R. Edelman, High-speed black blood imaging of vessel stenosis in the presence of pulsatile flow, J. Magn. Reson. Imaging. 2 (1992) 437-441. doi:10.1002/jmri.1880020413.

[3] L. Axel, Blood flow effects in magnetic resonance imaging, Am. J. Roentgenol. 143 (1984) 1157-1166. doi:10.2214/ajr.143.6.1157.

[4] A. Crombé, H.J. Brisse, P. Ledoux, L. Haddag-Miliani, A. Bouhamama, S. Taieb, F. Le Loarer, M. Kind, Alveolar soft-part sarcoma: can MRI help discriminating from other soft-tissue tumors? A study of the French sarcoma group, Eur. Radiol. (2018). doi:10.1007/s00330-018-5903-3.

[5] J.-F. Cui, H.-S. Chen, D.-P. Hao, J.-H. Liu, F. Hou, W.-J. Xu, Magnetic Resonance Features and Characteristic Vascular Pattern of Alveolar Soft-Part Sarcoma, Oncol. Res. Treat. 40 (2017) 580-585. doi:10.1159/000477443.

[6] D.T. Ginat, A. Bokhari, S. Bhatt, V. Dogra, Imaging features of solitary fibrous tumors, AJR Am. J. Roentgenol. 196 (2011) 487-495. doi:10.2214/AJR.10.4948.

[7] M.B. McCarville, S. Muzzafar, S.C. Kao, C.M. Coffin, D.M. Parham, J.R. Anderson, S.L. Spunt, Imaging features of alveolar soft-part sarcoma: a report from Children's Oncology Group Study ARST0332, AJR Am. J. Roentgenol. 203 (2014) 1345-1352. doi:10.2214/AJR.14.12462.

[8] S. Sood, A.D. Baheti, A.B. Shinagare, J.P. Jagannathan, J.L. Hornick, N.H. Ramaiya, S.H. Tirumani, Imaging features of primary and metastatic alveolar soft part sarcoma: single institute experience in 25 patients, Br. J. Radiol. 87 (2014) 20130719. doi:10.1259/bjr.20130719. 
[9] O.J. Wignall, E.C. Moskovic, K. Thway, J.M. Thomas, Solitary fibrous tumors of the soft tissues: review of the imaging and clinical features with histopathologic correlation, AJR Am. J. Roentgenol. 195 (2010) W55-62. doi:10.2214/AJR.09.3379.

[10] A.A. Azizi, C. Haberler, T. Czech, A. Gupper, D. Prayer, H. Breitschopf, T. Acker, I. Slavc, Vascular-endothelial-growth-factor (VEGF) expression and possible response to angiogenesis inhibitor bevacizumab in metastatic alveolar soft part sarcoma, Lancet Oncol. 7 (2006) 521-523. doi:10.1016/S1470-2045(06)70729-X.

[11] M.S. Park, V. Ravi, D.M. Araujo, Inhibiting the VEGF-VEGFR pathway in angiosarcoma, epithelioid hemangioendothelioma, and hemangiopericytoma/solitary fibrous tumor, Curr. Opin. Oncol. 22 (2010) 351-355. doi:10.1097/CCO.0b013e32833aaad4.

[12] L.H. Stockwin, D.T. Vistica, S. Kenney, D.S. Schrump, D.O. Butcher, M. Raffeld, R.H. Shoemaker, Gene expression profiling of alveolar soft-part sarcoma (ASPS), BMC Cancer. 9 (2009) 22. doi:10.1186/1471-2407-9-22.

[13] J.-A. Choi, K.H. Lee, W.S. Jun, M.G. Yi, S. Lee, H.S. Kang, Osseous metastasis from renal cell carcinoma: "flow-void" sign at MR imaging, Radiology. 228 (2003) 629-634. doi:10.1148/radiol.2283021153.

[14] C. Kuhnen, M. Lehnhardt, E. Tolnay, T. Muehlberger, P.M. Vogt, K.M. Müller, Patterns of expression and secretion of vascular endothelial growth factor in malignant soft-tissue tumours, J. Cancer Res. Clin. Oncol. 126 (2000) 219-225.

[15] A. Potti, A.K. Ganti, K. Tendulkar, K. Sholes, S. Chitajallu, M. Koch, S. Kargas, Determination of vascular endothelial growth factor (VEGF) overexpression in soft tissue sarcomas and the role of overexpression in leiomyosarcoma, J. Cancer Res. Clin. Oncol. 130 (2004) 52-56. doi:10.1007/s00432-003-0504-0.

[16] M. Trojani, G. Contesso, J.M. Coindre, J. Rouesse, N.B. Bui, A. de Mascarel, J.F. Goussot, M. David, F. Bonichon, C. Lagarde, Soft-tissue sarcomas of adults; study of pathological prognostic variables and definition of a histopathological grading system, Int. J. Cancer. 33 (1984) 37-42.

[17] J.M. Coindre, P. Terrier, N.B. Bui, F. Bonichon, F. Collin, V. Le Doussal, A.M. Mandard, M.O. Vilain, J. Jacquemier, H. Duplay, X. Sastre, C. Barlier, M. Henry-Amar, J. Macé-Lesech, G. Contesso, Prognostic factors in adult patients with locally controlled soft tissue sarcoma. A study of 546 patients from the French Federation of Cancer Centers Sarcoma Group, J. Clin. Oncol. Off. J. Am. Soc. Clin. Oncol. 14 (1996) 869877. doi:10.1200/JCO.1996.14.3.869.

[18] S.S. Yoon, N.H. Segal, A.B. Olshen, M.F. Brennan, S. Singer, Circulating angiogenic factor levels correlate with extent of disease and risk of recurrence in patients with soft 
tissue sarcoma, Ann. Oncol. Off. J. Eur. Soc. Med. Oncol. 15 (2004) 1261-1266. doi:10.1093/annonc/mdh309.

[19] T. Nakamura, A. Matsumine, T. Matsubara, K. Asanuma, Y. Yada, T. Hagi, A. Sudo, Infiltrative tumor growth patterns on magnetic resonance imaging associated with systemic inflammation and oncological outcome in patients with high-grade soft-tissue sarcoma, PloS One. 12 (2017) e0181787. doi:10.1371/journal.pone.0181787.

[20] F. Le Loarer, D. Pissaloux, S. Watson, C. Godfraind, L. Galmiche-Rolland, K. Silva, L. Mayeur, A. Italiano, A. Michot, G. Pierron, A. Vasiljevic, D. Ranchère-Vince, JM Coindre, F. Tirode. Clinicopathologic Features of CIC-NUTM1 Sarcomas, a New Molecular Variant of the Family of CIC-Fused Sarcomas. Am J Surg Pathol. 43 (2019) 268-276. doi: 10.1097/PAS.0000000000001187.

[21] D.A. Barbie, P. Tamayo, J.S. Boehm, S.Y. Kim, S.E. Moody, I.F. Dunn, A.C. Schinzel, P. Sandy, E. Meylan, C. Scholl, S. Fröhling, E.M. Chan, M.L. Sos, K. Michel, C. Mermel, S.J. Silver, B.A. Weir, J.H. Reiling, Q. Sheng, P.B. Gupta, R.C. Wadlow, H. Le, S. Hoersch, B.S. Wittner, S. Ramaswamy, D.M. Livingston, D.M. Sabatini, M. Meyerson, R.K. Thomas, E.S. Lander, J.P. Mesirov, D.E. Root, D.G. Gilliland, T. Jacks, W.C. Hahn, Systematic RNA interference reveals that oncogenic KRAS-driven cancers require TBK1, Nature. 462 (2009) 108-112. doi:10.1038/nature08460.

[22] A. Subramanian, P. Tamayo, V.K. Mootha, S. Mukherjee, B.L. Ebert, M.A. Gillette, A. Paulovich, S.L. Pomeroy, T.R. Golub, E.S. Lander, J.P. Mesirov, Gene set enrichment analysis: a knowledge-based approach for interpreting genome-wide expression profiles, Proc. Natl. Acad. Sci. U. S. A. 102 (2005) 15545-15550. doi:10.1073/pnas.0506580102.

[23] A. Liberzon, A. Subramanian, R. Pinchback, H. Thorvaldsdóttir, P. Tamayo, J.P. Mesirov, Molecular signatures database (MSigDB) 3.0, Bioinforma. Oxf. Engl. 27 (2011) 1739-1740. doi:10.1093/bioinformatics/btr260.

[24] J.R. Landis, G.G. Koch, The measurement of observer agreement for categorical data, Biometrics. 33 (1977) 159-174.

[25] H. Kato, M. Kanematsu, K. Mizuta, M. Aoki, B. Kuze, T. Ohno, K. Oshima, Y. Hirose, "Flow-void" sign at MR imaging: a rare finding of extracranial head and neck schwannomas, J. Magn. Reson. Imaging JMRI. 31 (2010) 703-705. doi:10.1002/jmri.22071.

[26] J.Y. Kim, J.H. Lee, J.G. Nam, S.H. Choi, Y.W. Seo, Y.K. Jeong, Value of tumor vessel sign in isolated circumscribed hypervascular abdominopelvic mesenchymal tumors on multidetector computed tomography, J. Comput. Assist. Tomogr. 38 (2014) 747-752. doi:10.1097/RCT.0000000000000099. 
[27] M.R. Robbin, M.D. Murphey, H.T. Temple, M.J. Kransdorf, J.J. Choi, Imaging of musculoskeletal fibromatosis, Radiogr. Rev. Publ. Radiol. Soc. N. Am. Inc. 21 (2001) 585-600. doi:10.1148/radiographics.21.3.g01ma21585.

[28] N. Griffin, N. Khan, J.M. Thomas, C. Fisher, E.C. Moskovic, The radiological manifestations of intramuscular haemangiomas in adults: magnetic resonance imaging, computed tomography and ultrasound appearances, Skeletal Radiol. 36 (2007) 10511059. doi:10.1007/s00256-007-0375-6.

[29] N. Setsu, A. Yoshida, F. Takahashi, H. Chuman, R. Kushima, Histological analysis suggests an invasion-independent metastatic mechanism in alveolar soft part sarcoma, Hum. Pathol. 45 (2014) 137-142. doi:10.1016/j.humpath.2013.07.045.

[30] A.J.F. Lazar, P. Das, D. Tuvin, B. Korchin, Q. Zhu, Z. Jin, C.L. Warneke, P.S. Zhang, V. Hernandez, D. Lopez-Terrada, P.W. Pisters, R.E. Pollock, D. Lev, Angiogenesispromoting gene patterns in alveolar soft part sarcoma, Clin. Cancer Res. Off. J. Am. Assoc. Cancer Res. 13 (2007) 7314-7321. doi:10.1158/1078-0432.CCR-07-0174.

[31] S. Stacchiotti, M. Tortoreto, G.G. Baldi, G. Grignani, A. Toss, G. Badalamenti, D. Cominetti, C. Morosi, A.P. Dei Tos, F. Festinese, E. Fumagalli, S. Provenzano, A. Gronchi, E. Pennacchioli, T. Negri, G.P. Dagrada, R.D. Spagnuolo, S. Pilotti, P.G. Casali, N. Zaffaroni, Preclinical and clinical evidence of activity of pazopanib in solitary fibrous tumour, Eur. J. Cancer Oxf. Engl. 1990. 50 (2014) 3021-3028. doi:10.1016/j.ejca.2014.09.004.

[32] S. Kummar, D. Allen, A. Monks, E.C. Polley, C.D. Hose, S.P. Ivy, I.B. Turkbey, S. Lawrence, R.J. Kinders, P. Choyke, R. Simon, S.M. Steinberg, J.H. Doroshow, L. Helman, Cediranib for metastatic alveolar soft part sarcoma, J. Clin. Oncol. Off. J. Am. Soc. Clin. Oncol. 31 (2013) 2296-2302. doi:10.1200/JCO.2012.47.4288.

[33] W.T.A. van der Graaf, J.-Y. Blay, S.P. Chawla, D.-W. Kim, B. Bui-Nguyen, P.G. Casali, P. Schöffski, M. Aglietta, A.P. Staddon, Y. Beppu, A. Le Cesne, H. Gelderblom, I.R. Judson, N. Araki, M. Ouali, S. Marreaud, R. Hodge, M.R. Dewji, C. Coens, G.D. Demetri, C.D. Fletcher, A.P. Dei Tos, P. Hohenberger, EORTC Soft Tissue and Bone Sarcoma Group, PALETTE study group, Pazopanib for metastatic soft-tissue sarcoma (PALETTE): a randomised, double-blind, placebo-controlled phase 3 trial, Lancet Lond. Engl. 379 (2012) 1879-1886. doi:10.1016/S0140-6736(12)60651-5.

[34] J. Hasan, R. Byers, G.C. Jayson, Intra-tumoural microvessel density in human solid tumours, Br. J. Cancer. 86 (2002) 1566-1577. doi:10.1038/sj.bjc.6600315.

[35] A. Shimizu, K.P. O’Brien, T. Sjöblom, K. Pietras, E. Buchdunger, V.P. Collins, C.H. Heldin, J.P. Dumanski, A. Ostman, The dermatofibrosarcoma protuberans-associated collagen type Ialpha1/platelet-derived growth factor (PDGF) B-chain fusion gene 
generates a transforming protein that is processed to functional PDGF-BB, Cancer Res. 59 (1999) 3719-3723.

[36] K. Yonemori, K. Tsuta, M. Ando, A. Hirakawa, Y. Hatanaka, Y. Matsuno, H. Chuman, N. Yamazaki, Y. Fujiwara, T. Hasegawa, Contrasting prognostic implications of platelet-derived growth factor receptor- $\beta$ and vascular endothelial growth factor receptor-2 in patients with angiosarcoma, Ann. Surg. Oncol. 18 (2011) 2841-2850. doi:10.1245/s10434-011-1640-4. 
A.1 Coro STIR T2-WI

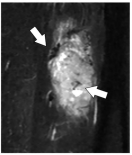

B.1

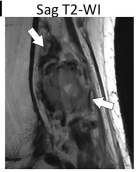

Ax T2-WI

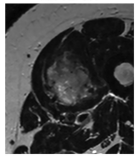

Ax Fat Sat T2-WI

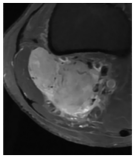

A. 2

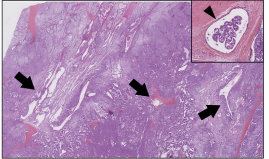

B. 2

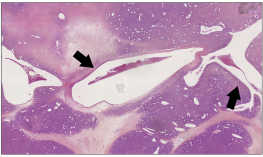


A Coro STIR T2-WI

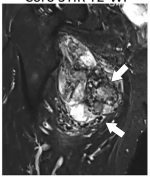

Ax T2-WI

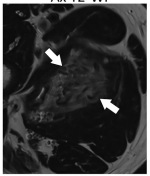

B

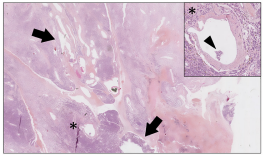

C

HALLMARK_ANGIOGENESIS

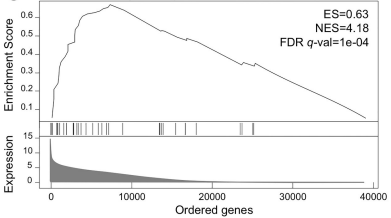




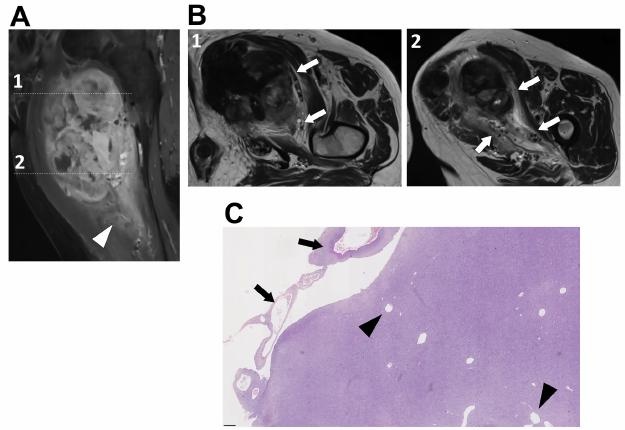



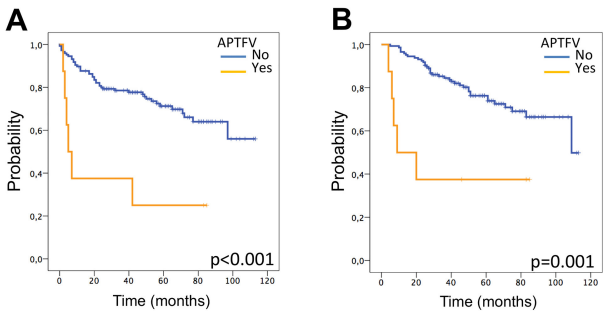
Table 1. Epidemiological characteristics of the study population

\begin{tabular}{lc}
\hline Characteristics & Patients \\
\hline Age (years) & \\
Median (range) & $61(15-92)$ \\
Gender & \\
Male & $88 / 157(56.1)$ \\
Female & $69 / 157(43.9)$ \\
Histotype & \\
Undifferentiated pleomorphic sarcoma & $39 / 157(24.8)$ \\
Undifferentiated sarcoma - others & $15 / 157(5.7)$ \\
Myxofibrosarcoma & $24 / 157(15.3)$ \\
Liposarcoma - dedifferentiated & $13 / 157(8.3)$ \\
Lipoarcoma - pleomorphic & $7 / 157(4.5)$ \\
Liposarcoma - myxoid / round cells & $11 / 157(7)$ \\
Leiomyosarcoma & $16 / 157(10.2)$ \\
Synovial sarcoma & $5 / 157(3.2)$ \\
Low grade fibromyxoid sarcoma & $6 / 157(3.8)$ \\
Extraskeletal myxoid chondrosarcoma & $5 / 157(3.2)$ \\
Rhabdomyosarcoma & $4 / 157(2.5)$ \\
Malignant peripheral nerve sheath tumor & $4 / 157(2.5)$ \\
Malignant solitary fibrous tumor & $2 / 157(1.3)$ \\
Alveolar soft-part sarcoma & $1 / 157(0.6)$ \\
Location & \\
Upper limb & $13 / 157(8.3)$ \\
Shoulder girdle & $13 / 157(8.3)$ \\
Trunk wall & $19 / 157(12.1)$ \\
Pelvic girdle & $9 / 157(5.7)$ \\
Lower limb & $103 / 157(65.6)$ \\
Depth & \\
Superficial & $11 / 157(7)$ \\
Deep and superficial & $55 / 157(35)$ \\
Deep & $91 / 157(58)$ \\
Longest diameter (mm) & \\
Mean (+/- standard deviation) & $88.7+/-56$ \\
Median (range) & $74(10-192)$ \\
FNCLCC grade & \\
grade I & $21 / 157(13.4)$ \\
grade II & $48 / 157(30.6)$ \\
grade III & $88 / 157(56.1)$ \\
\hline &
\end{tabular}

NOTE. Data are number of patients with percentage in parentheses, except for age (given as median and range, in years) and longest diameter (given as mean and standard deviation, as well as median and range, in $\mathrm{mm}$ ).

Abbreviations : FNCLCC : French 'Federation Nationale des Centres de Lutte Contre le Cancer' grading system.

$\S$ : Other undifferentiated sarcomas (US) are: fusiform US (3/157, 1.9\%), epitheloid US $(3 / 157,1.9 \%)$, clear cells US $(1 / 157,0.6 \%)$, round cells US $(1 / 157,0.6 \%)$, not otherwise specified US $(7 / 157,4.5 \%)$ 
Table 2. Association between abnormal vascularization on conventional MRI and histological grading

\begin{tabular}{lccccc}
\hline Characteristics & Grade I & Grade II & Grade III & $\begin{array}{c}\text { p-value } \\
\text { (I vs II vs III) }\end{array}$ & $\begin{array}{c}\text { p-value } \\
\text { (I vs II-III) }\end{array}$ \\
\hline Flow Voids & $19 / 21(90.5)$ & $39 / 48(81.3)$ & $70 / 88(80.7)$ & 0.509 & 0.370 \\
$\quad$ Absent & $2 / 21(9.5)$ & $9 / 48(18.8)$ & $18 / 88(19.3)$ & & \\
$\quad$ Present & & & & \\
Flow Voids - distribution & $19 / 21(90.5)$ & $39 / 48(81.3)$ & $70 / 88(80.7)$ & 0.267 & 0.494 \\
$\quad$ None & $0 / 21(0)$ & $1 / 48(2.1)$ & $8 / 88(8)$ & & \\
$\quad$ Peri-tumoral & $1 / 21(4.8)$ & $3 / 48(6.5)$ & $1 / 88(1.1)$ & & \\
$\quad$ Intra-tumoral & $1 / 21(4.8)$ & $5 / 48(10.4)$ & $9 / 88(10.2)$ & & \\
$\quad$ Intra- and peri-tumoral & & & & & \\
Flow Voids - count & $19 / 21(90.5)$ & $39 / 48(81.3)$ & $70 / 88(79.5)$ & 0.860 & \\
0 & $2 / 21(9.5)$ & $6 / 48(12.5)$ & $11 / 88(12.5)$ & & \\
$1-5$ & $0 / 21(0)$ & $3 / 48(6.5)$ & $6 / 88(6.8)$ & & \\
$6-10$ & $0 / 21(0)$ & $0 / 48(0)$ & $1 / 88(1.1)$ & & \\
$>10$ & & & & & \\
Flow Voids - surface & $20 / 21(95.2)$ & $42 / 48(87.5)$ & $71 / 88(80.7)$ & 0.467 & \\
$0 \%$ & $1 / 21(4.8)$ & $6 / 48(12.5)$ & $16 / 88(18.2)$ & & \\
$1-25 \%$ & $0 / 21(0)$ & $0 / 48(0)$ & $1 / 88(1.1)$ & & \\
$>25 \%$ & $3+/-1.4(2-4)$ & $2.1+/-0.8(1-3)$ & $3.1+/-2.3(1-10)$ & 0.469 & 0.880 \\
Flow Void - diameter & &
\end{tabular}

NOTE. Data are number of patients with percentage in parentheses.

Grade corresponds to the French Federation Nationale des Centres de Lutte Contre le Cancer grading system 
Table 3. Inter-observer agreement of the features evaluating the vascularization of STS on conventional MRIs.

\begin{tabular}{lc}
\hline Characteristics & Inter-observer agreement \\
\hline Flow-voids - count (1) & $0.76(0.67-0.85)$ \\
Flow-voids - surface\$ (1) & $0.51(0.50-0.52)$ \\
Flow-voids - distribution (1) & $0.53(0.12-0.95)$ \\
Flow-voids - maximal diameter (2) & $0.82(0.76-0.87)$ \\
\hline
\end{tabular}

NOTE. Data are inter-observer agreements with 95\% confidence interval in parentheses. Interobserver agreements were evaluated with: (1) weighted Kappa for ordinal variables, and (2) inter-class correlation coefficient for numeric variables.

$\S$ : corresponds to the tumor circumference covered by abnormal peri-tumoral flow-voids. 
Table 4. Univariate survival analysis

\begin{tabular}{|c|c|c|c|c|c|c|c|c|c|c|c|c|}
\hline \multirow[b]{2}{*}{ Characteristics } & \multicolumn{4}{|c|}{ Metastasis-Free Survival } & \multicolumn{4}{|c|}{ Local-Relapse-Free Survival } & \multicolumn{4}{|c|}{ Overall Survival } \\
\hline & $\begin{array}{c}\text { No. of } \\
\text { patients }\end{array}$ & $\begin{array}{l}\text { No. of } \\
\text { events }\end{array}$ & Survival & $p$-value & $\begin{array}{c}\text { No. of } \\
\text { patients }\end{array}$ & $\begin{array}{l}\text { No. of } \\
\text { events }\end{array}$ & Survival & p-value & $\begin{array}{c}\text { No. of } \\
\text { patients }\end{array}$ & $\begin{array}{l}\text { No. of } \\
\text { events }\end{array}$ & Survival & p-value \\
\hline \multicolumn{13}{|l|}{ Flow Voids } \\
\hline Absent & 125 & 38 & $81(72-89)$ & & 125 & 30 & $88(80-96)$ & 0.430 & 125 & 31 & $89(82-96)$ & 0,225 \\
\hline Present & 29 & 11 & $74(56-92)$ & 0.478 & 29 & 5 & $87(75-99)$ & & 29 & 12 & $74(59-92)$ & \\
\hline \multicolumn{13}{|l|}{ Flow Voids - distribution } \\
\hline None & 125 & 38 & - & $0.014^{\star}$ & 125 & 30 & - & 0.059 & 125 & 31 & $89(82-96)$ & $0.039^{*}$ \\
\hline Intra-tumoral & 5 & 0 & - & & 5 & 3 & - & & 5 & 1 & $93(59-127)$ & \\
\hline Intra- and peritumoral & 15 & 5 & - & & 15 & 0 & - & & 15 & 6 & $76(62-91)$ & \\
\hline \multicolumn{13}{|l|}{ APTFV } \\
\hline No & 145 & 43 & $83(75-90)$ & $0.003^{\star \star}$ & 145 & 33 & $89(82-96)$ & 0.665 & 145 & 38 & $89(82-95)$ & $0.006^{*}$ \\
\hline Yes & 9 & 6 & $35(11-59)$ & & 9 & 2 & $67(45-89)$ & & 9 & 5 & $43(18-68)$ & \\
\hline \multicolumn{13}{|l|}{ Flow Voids - count } \\
\hline 0 & 125 & 38 & - & 0.610 & 125 & 30 & - & 0.687 & 125 & 31 & - & 0.106 \\
\hline $0-5$ & 19 & 8 & - & & 19 & 4 & - & & 19 & 9 & - & \\
\hline$>10$ & 1 & 0 & - & & 1 & 0 & - & & 1 & 0 & - & \\
\hline \multicolumn{13}{|l|}{ Flow Voids - surface§ } \\
\hline $0 \%$ & 130 & 38 & - & 0.164 & 130 & 33 & - & 0.231 & 130 & 32 & - & 0.071 \\
\hline $1-25 \%$ & 23 & 11 & - & & 23 & 2 & - & & 23 & 11 & - & \\
\hline$>25 \%$ & 1 & 0 & - & & 1 & 0 & - & & 1 & 0 & - & \\
\hline
\end{tabular}

NOTE. Abbreviations : APTFV : abnormal perit-tumoral flow-voids, no.: number, HR: hazard ratio, CI95\%: 95\% confidence interval

Events correspond to: (i) a metastatic relapse for metastasis-free survival, (ii) a local relapse for local relapse-free survival, and (iii) death for overall survival. Survivals correspond to mean survival in months and are given with 95\% confidence interval.

$\S:$ corresponds to the tumor circumference covered by flow-voids.

$*: \mathrm{p}<0.05, * *: \mathrm{p}<0.005, * * *: \mathrm{p}<0.001$ 